\title{
MODELAGEM DA CAPACIDADE DE SUPORTE DE CARGA E QUANTIFICAÇÃO DOS EFEITOS DAS OPERAÇÕES MECANIZADAS EM UM LATOSSOLO AMARELO CULTIVADO COM CAFEEIROS ${ }^{(1)}$
}

\author{
Arystides Resende Silva ${ }^{(2)}$, Moacir de Souza Dias Junior ${ }^{(3)}$, Paulo \\ Tácito Gontijo Guimarães ${ }^{(4)} \&$ Cezar Franciso Araújo Júnior ${ }^{(5)}$
}

\begin{abstract}
RESUMO
O Estado de Minas Gerais é o maior produtor de café do Brasil, com mais de $50 \%$ da produção total, sendo grande parte da área cultivada originalmente ocupada por vegetação de cerrado. Essa região é destaque na cafeicultura em razão da alta tecnologia empregada na mecanização, que submete o solo ao tráfego de máquinas, tornando preocupante a possibilidade de disseminação da compactação do solo. Objetivou-se com este estudo: (a) propor modelos de capacidade de suporte de carga de um Latossolo Amarelo distrófico típico (LAd) cultivado com cafeeiro em função da pressão de preconsolidação e da umidade, (b) determinar, com o uso destes modelos, em três profundidades e em duas épocas de amostragem, a influência das operações de manejo na estrutura do Latossolo Amarelo nas estações: seca e chuvosa. As amostragens foram realizadas em três camadas (0-3, 10-13 e 25-28 cm), sendo 30 amostras coletadas no local onde não houve tráfego e 10 amostras na linha de tráfego para cada equipamento, coletadas nos meses de agosto de 2002 e março de 2003 para quantificar seu efeito na estrutura
\end{abstract}

\footnotetext{
(1) Parte da Tese de Mestrado do primeiro autor, apresentado ao Departamento de Ciência do Solo - DCS/UFLA. Projeto financia pelo Consórcio Brasileiro de Pesquisa e Desenvolvimento do Café e pela CAPES. Recebido para publicação em outubro de 2003 e aprovado em fevereiro de 2006.

(2) Engenheiro Florestal, Doutorando do Departamento de Ciência do Solo, Universidade Federal de Lavras - UFLA. Caixa Postal 3037, CEP 37200-000 Lavras (MG). Bolsista CAPES. E-mail: arystidesrs@yahoo.com.br

(3) Professor do Departamento de Ciência do Solo, UFLA. Bolsistas CNPq. E-mail: msouzadj@ufla.br

(4) Pesquisador da Empresa de Pesquisa Agropecuária de Minas Gerais - EPAMIG. Campus UFLA, Caixa Postal 176, CEP 37200000 Lavras (MG). E-mail: paulotgg@ufla.br

(5) Aluno de Iniciação Cientifica. Departamento de Ciência do Solo, UFLA. E-mail: cfaj@bol.com.br
} 
do solo nas duas épocas: seca e chuvosa, respectivamente. As amostras indeformadas foram utilizadas nos ensaios de compressão uniaxial. Foram determinados também granulometria, matéria orgânica, densidade de partículas, limite de contração e limite de plasticidade do solo. O modelo de capacidade de suporte de carga do LAd é expresso pela equação $\sigma_{\mathrm{p}}=10^{(2,72-1,17 \mathrm{U})}$, em que $\sigma_{\mathrm{p}}$ significa a pressão de preconsolidação e U a umidade. Os efeitos das operações mecanizadas na lavoura cafeeira foram quantificados com o modelo de capacidade de suporte de carga e com o seu uso foi possível quantificar a influência das operações de manejo na estrutura do Latossolo Amarelo distrófico típico.

Termos de indexação: compactação do solo, café, manejo do solo, pressão de preconsolidação, tráfego.

\title{
SUMMARY: MODELLING THE LOAD SUPPORT CAPACITY AND QUANTIFICATION OF MECHANIZED OPERATION EFFECTS ONA YELLOW LATOSOL UNDER COFFEE PLANTATIONS
}

\begin{abstract}
Minas Gerais state is the largest coffee producer in Brazil. The state contributes with over $50 \%$ of the total production, which is for the most part cultivated in the Cerrado region. This region is outstanding in coffee production due to the advanced technology in mechanization. Consequently, the machine traffic that the soils are subjected to causes concern because of the possibility of widespread soil compaction. Our objectives were (a) to propose load support capacity models to a typical dystrophic Yellow Latosol (LAd) cultivated with coffee as a function of preconsolidation pressure and moisture content; (b) to determine, through the use of this model, the influence of soil management operations on the soil structure at three depths, in the dry and rainy seasons. Soil samples were taken from three depths ( 0 to 3, 10 to 13 and 25 to $28 \mathrm{~cm}$ ); thirty samples were collected where there was no traffic and ten samples collected in the traffic line of each equipment, in August 2002 and March 2003, respectively, to quantify its effects on the soil structure in the rainy and in the dry seasons. The undisturbed soil samples were used in the uniaxial compression test. Texture, organic matter, particle density, shrinkage limit, and plasticity limit were also determined. The load support capacity model of the LAd is a function of the preconsolidation pressure and moisture content and is expressed by the equation $\sigma_{p}=10^{(2.72-1.17 U)}$ where $\sigma_{p}$ means preconsolidation pressure and U means humidity. The effects of the mechanized operations in the coffee plantation were quantified by the load support capacity model and through its use it was possible to quantify the influence of the management operations in the Yellow Latosol structure.
\end{abstract}

Index terms: soil compaction, coffee, modelling, preconsolidation pressure, traffic.

\section{INTRODUÇÃO}

A cultura do cafeeiro no Brasil destaca-se por sua grande área de cultivo e por se tratar de um produto de exportação, de grande importância econômica. Atualmente, o Estado de Minas Gerais é o maior produtor do Brasil, com mais de $50 \%$ da produção total, sendo grande parte da área cultivada originalmente ocupada por vegetação de cerrado. Essa região é lugar de destaque pelas excelentes condições de topografia e clima favoráveis à cafeicultura (Teodoro et al., 2003).

Nas regiões do Triângulo Mineiro e Alto Paranaíba, a cafeicultura tem-se desenvolvido de forma acelerada em razão da alta tecnologia aplicada, sendo mecanizada em todas as suas etapas, desde a fase de instalação até à fase de produção.

A intensa mecanização aplicada aos solos da Região dos cerrados tem comprometido suas propriedades físicas, tais como: a permeabilidade à água e ao ar, água disponível, a densidade do solo e a erodibilidade (Guimarães, 2000; Ishaq et al., 2001; Gysi, 2001; Pytka, 2001; Canillas \& Salokhe, 2002; Williamson \& Nielsen, 2003, Oliveira et al., 2003). O uso de máquinas agrícolas nas várias etapas do processo de produção em condições inadequadas de umidade (Dias Junior \& Pierce, 1996; Novais \& Smyth, 1999; Imhoff et al., 2001; Servadio et al., 2001; Silva et al., 2002; Chamen et al., 2003) tem sido o principal responsável por acarretar a 
degradação da estrutura do solo, diminuindo seu potencial produtivo. Esta degradação pode estar associada à compactação do solo (Ribeiro, 1999; Çarman, 2002; Chamen et al., 2003).

Vários são os efeitos que a compactação do solo pode causar às plantas: a demora na emergência, a diminuição no tamanho, a presença de folhas com coloração não-característica, sistema radicular superficial e raízes mal formadas (Mantovani, 1987). No solo, a compactação ocasiona aumento da sua densidade e da resistência mecânica (Dias Junior et al., 1999; Arvidsson, 2001; Ishaq et al., 2001; Nevens \& Reheul, 2003; Williamson \& Neilsen, 2003); diminuição na porosidade total, tamanho e continuidade dos poros (Dias Junior, 2000; Servadio et al., 2001); redução na absorção de nutrientes, infiltração e redistribuição de água (Arvidsson, 2001; Ishaq et al., 2001); redução da condutividade hidráulica (Arvidsson, 2001), redução das trocas gasosas (Gysi, 2001) e aumento da capacidade de suporte de carga do solo (Dias Junior, 1994; Dias Junior \& Pierce, 1996; Kondo, 1998; Silva et al., 1999; Dias Junior, 2000; Miranda et al., 2003).

O desenvolvimento da cafeicultura está relacionado com o uso de máquinas agrícolas que podem causar compactação do solo (Larson et al., 1989; Dias Junior \& Pierce, 1996; Dias Junior, 2000), alterando o meio onde o sistema radicular se desenvolve (Gysi, 2001). Portanto, o tráfego em condições inadequadas de umidade em áreas cultivadas com cafeeiros tem-se tornado preocupante em decorrência da compactação causada pelas máquinas ao longo dos anos, que pode levar à redução da produtividade. Assim, estudos que visem à identificação, quantificação e minimização dos efeitos causados pelo manejo da cultura sobre o solo são importantes para adaptar, de forma condizente, o manejo, tendo em vista o desenvolvimento de uma cafeicultura sustentável (Miranda et al., 2003).

Dentro desse contexto, procurou-se incentivar estudos que contemplem o desenvolvimento de modelos de capacidade de suporte de carga das diferentes classes de solo, em face da história de seu manejo e da umidade (Dias Junior, 1994). Estudos recentes têm utilizado modelos para estimar a capacidade de suporte de carga dos solos, quantificando os níveis de pressões que podem ser aplicados aos solos para evitar que a compactação ocorra, tais como os de Dias Junior et al. (1999); Miranda et al. (2003); Oliveira et al. (2003), e o monitoramento das práticas de manejo, visando evitar ou minimizar a compactação dos solos.

Diante dessas considerações, objetivou-se com este estudo: (a) propor modelos de capacidade de suporte de carga de um Latossolo Amarelo cultivado com cafeeiro, relacionada com a pressão de preconsolidação e com a umidade; (b) determinar, com o uso dos modelos propostos, a influência das operações de manejo na estrutura do Latossolo
Amarelo em três camadas e em duas épocas de amostragem - estações seca e chuvosa.

\section{MATERIAL E MÉTODOS}

Este estudo foi realizado na Fazenda Experimental da EPAMIG (Empresa de Pesquisa Agropecuária de Minas Gerais), em Patrocínio (MG), localizada na região do Alto Paranaíba, cuja sede tem as seguintes coordenadas: $18^{\circ} 57^{\prime} 00 " \mathrm{~S}$ e $47^{\circ} 00^{\prime} 00^{\prime}$ W de Greenwich, e altitude de $934 \mathrm{~m}$.

O clima é o tropical chuvoso, com temperatura média anual de $21,8^{\circ} \mathrm{C}$, precipitação pluvial média anual de $1.372 \mathrm{~mm}$, distribuída no período de outubro a março, apresentando períodos bem definidos de seca e de chuva e baixa umidade relativa do ar. O relevo é suave ondulado em grandes extensões e a classe de solo predominante é a de Latossolos (Borges et al., 2001). O solo da área estudada, de acordo com os critérios adotados no Sistema Brasileiro de Classificação de Solo (Embrapa, 1999), foi classificado como Latossolo Amarelo distrófico típico (LAd) textura argilosa.

Em 1977, foi selecionado um talhão em área originalmente de cerrado, desmatada e seguida de aração com grade Rome. Esta área permaneceu em pousio até 2002. A área do talhão era de $1.800 \mathrm{~m}^{2}$, tendo $36 \mathrm{~m}$ de largura e $50 \mathrm{~m}$ de comprimento. Por ocasião do experimento, essa área foi limpa manualmente, retirando-se toda a vegetação para a instalação e realização do experimento.

O estudo utilizou dois tipos de amostragens: (a) amostras indeformadas, coletadas no local onde não houve tráfego, utilizadas no desenvolvimento dos modelos de capacidade de suporte de carga para o Latossolo Amarelo distrófico típico, e (b) amostras indeformadas, coletadas na linha de tráfego na época seca, em agosto de 2002, e na época chuvosa, em março de 2003, para verificar os efeitos das operações mecanizadas do manejo da lavoura de café sobre a estrutura do solo.

A precipitação pluvial do mês de agosto de 2002 foi de $1,8 \mathrm{~mm}$. As operações executadas na época chuvosa foram realizadas entre os meses de novembro de 2002 a março de 2003, e as amostras foram coletadas no mês de março de 2003. As precipitações pluviais foram de 147,2, 306,2, 347,2, 176,6 e $11,6 \mathrm{~mm}$, para os meses de novembro, dezembro, janeiro, fevereiro e março (este valor se refere à precipitação até o dia 10 de março de 2003, dia da última operação e amostragem), respectivamente.

Para a elaboração do modelo de capacidade de suporte de carga, foram coletadas 30 amostras indeformadas para cada camada $(0-3,10-13$ e $25-$ $28 \mathrm{~cm}$ ), usando-se um amostrador tipo Uhland com anel volumétrico de $6,40 \mathrm{~cm}$ de diâmetro e $2,54 \mathrm{~cm}$ 
de altura. As amostras foram parafinadas no campo para evitar a ocorrência de alterações na umidade a partir do momento da coleta até à realização do ensaio de compressão uniaxial no laboratório.

Estas amostras foram submetidas ao ensaio de compressão uniaxial de acordo com o método proposto por Bowles (1986), modificado por Dias Junior (1994).

Para realizar os ensaios de compressão uniaxial, as amostras foram previamente saturadas e depois secas ao ar no laboratório até se obterem as faixas de umidade de 0,05 a 0,63 kg kg-1. Após a obtenção dessas umidades, as amostras foram submetidas ao ensaio de compressão uniaxial, usando um consolidômetro da marca Boart Longyear, por meio do qual as pressões foram aplicadas, utilizando-se ar comprimido. As pressões aplicadas a cada amostra obedeceram à seguinte ordem: 25, 50, 100, $200,400,800,1.600 \mathrm{kPa}$. Cada pressão foi aplicada até que $90 \%$ da deformação máxima fosse atingida (Taylor, 1971) e, após essa condição ser atingida, aplicou-se uma nova pressão.

Após o ensaio de compressão uniaxial, as pressões de preconsolidação $\left(\sigma_{p}\right)$ foram obtidas na curva de compressão do solo de acordo com Dias Junior \& Pierce (1995). As pressões de preconsolidação foram marcadas de acordo com a umidade, utilizando o software Sigma Plot 8.0 (Jandel Scientific, P.O. Box 7005, San Rafael, CA, USA), bem como foram obtidas as equações matemáticas correspondentes aos modelos de capacidade de suporte de carga com os respectivos intervalos de confiança da população a $95 \%$.

Para os ensaios nas épocas seca e chuvosa, amostras indeformadas foram coletadas nas linhas de tráfego das máquinas e equipamentos após as seguintes operações:

Operação 1 - Colhedora modelo KTR APG/SR (Série 2000, marca JATO, massa $5.280 \mathrm{~kg}$ ). Trafegou uma vez na estação seca sendo tracionada pelo trator marca Massey Ferguson 275 (potência de $53 \mathrm{~kW}$ no motor (72 cv), pneus traseiros R1 18.4-30 e dianteiros 7.50-16, massa de $4.393 \mathrm{~kg}$ ).

Operação 2 - Pulverizador modelo Arbus 2000 (marca Jacto, capacidade de $2.600 \mathrm{~kg}$ quando completamente carregado). Trafegou uma vez na estação seca e três vezes na estação chuvosa, sendo tracionado pelo trator marca Massey Ferguson, modelo 265 (potência de $45 \mathrm{~kW}$ no motor $(61 \mathrm{cv}$ ), pneus traseiros R1 18.4-30 e dianteiros R1 7.50-16, massa $3.488 \mathrm{~kg}$ ).

Operação 3 - Roçadora modelo RC2 1500 (marca tatu, massa $388 \mathrm{~kg}$ ). Trafegou quatro vezes na estação seca, sendo tracionada pelo trator marca Massey Ferguson (modelo 50X, massa de $3.020 \mathrm{~kg}$ ). Trafegou também três vezes na estação chuvosa, sendo uma vez tracionada pelo trator Massey Ferguson 50X e as outras duas vezes pelo trator Massey Ferguson 265, (massa 3.488 kg).
Operação 4 - Granuladora modelo TURBO CK2AG/110 (marca Kamaq, quando completamente carregada apresenta massa de $340 \mathrm{~kg}$ ). Trafegou duas vezes na estação chuvosa, tracionada pelo trator Massey Fergusson (50X, massa de 3.020 kg).

Operação 5 - Adubadora modelo Fertinox; quando completamente carregada, tem capacidade para $600 \mathrm{~kg}$. Trafegou três vezes na estação chuvosa, tracionada pelo trator Massey Fergusson 50X, (massa de $3.020 \mathrm{~kg}$ ).

Operação 6 - Aplicador de herbicida modelo PH 400 (marca Arbus, apresenta $510 \mathrm{~kg}$ de massa, quando completamente carregado. Trafegou uma vez na estação chuvosa, tracionado pelo trator Massey Ferguson 265 (massa de 3.488 kg).

Operação 7 - Avaliou o efeito cumulativo do tráfego de todos estes equipamentos mais o efeito do tráfego da colhedora, visto que, graças à sua maior bitola, a linha de tráfego não coincidiu com a dos outros equipamentos.

Os equipamentos trafegaram em local especificado para cada um, em uma distância de $50 \mathrm{~m}$ de comprimento, e com distância de $2 \mathrm{~m}$ entre a linha de tráfego de cada equipamento.

Para determinar a influência das operações de manejo na estrutura do solo, na estação seca, foram coletadas 10 amostras indeformadas por camada para cada equipamento e mais 10 amostras por camada na linha de tráfego onde houve o efeito cumulativo do tráfego de todos os equipamentos e mais três amostras por camada na linha de tráfego da colhedora, por não ter coincidido, graças à sua maior bitola, sua linha de tráfego com a dos outros equipamentos. O número total de amostras coletadas foi de 129 amostras [10 amostras x três camadas x quatro operações (operações 1, 2, 3 e 7) + três amostras x três camadas].

No laboratório, os ensaios de compressão uniaxial foram realizados em amostras coletadas na época seca. Obtiveram-se, então, os valores das pressões de preconsolidação que foram inseridos nos modelos de capacidade de suporte de carga, determinandose o efeito dos equipamentos sobre a estrutura do Latossolo Amarelo distrófico típico (LAd) na estação seca.

Para determinar a influência das operações de manejo na estrutura do solo, na estação chuvosa, foram coletadas 10 amostras indeformadas por camada para cada equipamento estudado, mais 10 amostras por camada na linha de tráfego onde houve o efeito cumulativo do tráfego de todos equipamentos. O número total de amostras foi de 180 [10 amostras $\mathrm{x}$ três camadas $\mathrm{x}$ seis operações (operações 2, 3, 4, 5, 6 e 7)].

No laboratório, os ensaios de compressão uniaxial foram realizados nas amostras coletadas na época chuvosa. 
Para analisar os efeitos das operações 1 a 7 sobre a estrutura do LAd, as pressões de preconsolidação determinadas após estas operações foram inseridas no modelo de capacidade de suporte (Figuras 3 a 6). Três regiões foram consideradas: uma acima do limite de confiança superior, considerada como a região na qual a compactação do solo já ocorreu; outra situada entre os limites de confiança, considerada como região na qual existe tendência de ocorrer a compactação, e uma situada abaixo do limite de confiança inferior, considerada como a região na qual não existe compactação do solo (Dias Junior, 2003).

As amostras deformadas foram obtidas pela coleta de solo das porções superiores e inferiores dos anéis de amostragem, nas quais foram determinados os teores de matéria orgânica (Raij \& Quaggio, 1983), granulometria (Day, 1986), densidade de partículas (Blake \& Hartge, 1986), limite de plasticidade (Sowers, 1965) e limite de contração Bowles (1986) (Quadro 1).

Análises de regressões foram efetuadas com o uso do software Sigma Plot 8.0 (Jandel Scientific) e as comparações das regressões foram feitas utilizando o procedimento descrito em Snedecor \& Cochran (1989).

Os resultados das análises das características físicas do solo (Quadro 1) foram submetidos à análise de variância, e a comparação das médias das profundidades, ao teste de Scott-Knott $(p<0,05)$.

\section{RESULTADOS E DISCUSSÃO}

Os valores dos parâmetros estimados "a" e "b" do modelo de capacidade de suporte de carga $\left[\sigma_{\mathrm{p}}=10^{(\mathrm{a}+\mathrm{bU})}\right]$ do Latossolo Amarelo distrófico típico (LAd) sob vegetação de cerrado, nas camadas de 0-3, 10-13 e 25-28 cm (Figura 1), variaram de 2,72 a 2,74 e de $-1,05$ a $-1,26$, respectivamente. Os coeficientes de determinação $\left(R^{2}\right)$ variaram de 0,82 a 0,88 , sendo todos significativos a $1 \%$ (Figura 1$)$.
Os modelos de capacidade de suporte de carga não foram estatisticamente diferentes para as três camadas (Quadro 2), o que pode ser justificado pelo fato de que, em 1977, ter ocorrido uma aração, logo após o desmatamento, proporcionando uma homogeneização das camadas em relação à sua capacidade de suporte de carga.

Decorrente dessa não-significância, uma nova equação de regressão foi ajustada, considerando todos os valores de pressão de preconsolidação e de umidade, obtendo-se um único modelo de capacidade de suporte de carga, $\sigma_{\mathrm{p}}=10^{(2,72-1,17 \mathrm{U})}$, com $\mathrm{R}^{2}=0,85^{* *}$ $(\mathrm{n}=89)$, para as três camadas do Latossolo Amarelo distrófico (Figura 2).

A capacidade de suporte de carga do LAd na zona de friabilidade, que corresponde à faixa de umidade adequada para o preparo do solo (Hillel, 1982), variou de 204 a $210 \mathrm{kPa}$ para a camada de $0-3 \mathrm{~cm}$; de 179 a $222 \mathrm{kPa}$, para a camada de $10-13 \mathrm{~cm}$, e de 165 a $234 \mathrm{kPa}$, para a camada de $25-28 \mathrm{~cm}$. De acordo com Carpenedo (1994), as pressões médias normalmente aplicadas à superfície do solo pelas máquinas são próximas de $200 \mathrm{kPa}$. Assim, o preparo deste solo em umidade próxima ao limite de plasticidade (Figuras 3 a 6 e Quadro 1) pode causar compactação, principalmente nas camadas de $10-13$ e de $25-28 \mathrm{~cm}$ pelo fato de as $\sigma_{\mathrm{p}}$ do solo

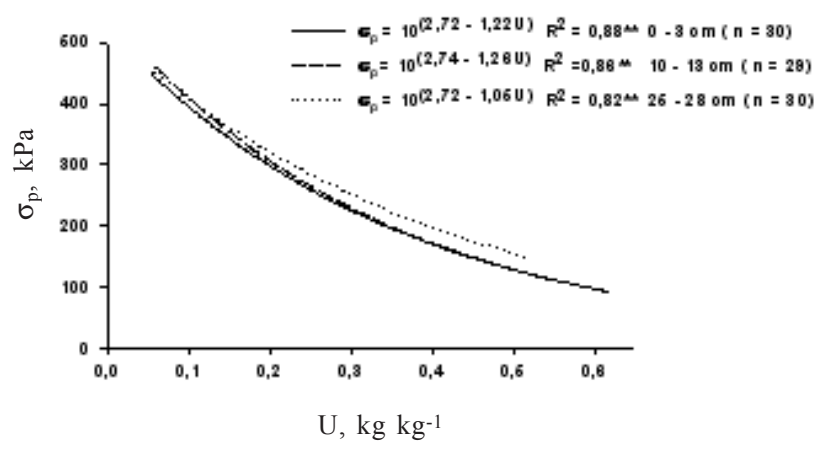

Figura 1. Modelos da capacidade de suporte de carga para o Latossolo Amarelo distrófico típico nas camadas de 0-3, 10-13 e 25-28 cm.

Quadro 1. Granulometria, teores de matéria orgânica (MO), densidade de partículas (Dp), limite de plasticidade (LP) e limite de contração (LC) de um Latossolo Amarelo distrófico típico (LAd), obtidos em três camadas na área em estudo

\begin{tabular}{|c|c|c|c|c|c|c|c|}
\hline Camada & Argila & Areia & Silte & MO & Dp & $\mathbf{L P}$ & LC \\
\hline $\mathrm{cm}$ & 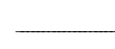 & 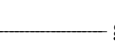 & & - & $\mathrm{g} \mathrm{cm}^{-3}$ & 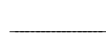 & 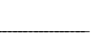 \\
\hline $0-3$ & $506 \mathrm{a}$ & $139 a$ & $355 \mathrm{a}$ & $31 \mathrm{a}$ & $2,55 \mathrm{a}$ & $0,35 \mathrm{a}$ & $0,34 \mathrm{a}$ \\
\hline $10-13$ & $531 \mathrm{~b}$ & $142 \mathrm{a}$ & $327 \mathrm{~b}$ & $27 \mathrm{~b}$ & $2,58 \mathrm{~b}$ & $0,40 \mathrm{~b}$ & $0,32 \mathrm{a}$ \\
\hline $25-28$ & $544 \mathrm{~b}$ & $149 \mathrm{~b}$ & $307 \mathrm{~b}$ & $25 \mathrm{~b}$ & $2,62 \mathrm{c}$ & $0,43 \mathrm{~b}$ & $0,30 \mathrm{a}$ \\
\hline
\end{tabular}

Médias, nas colunas, com a mesma letra não diferem entre si pelo teste de Scott-Knott a 5 \%. 
Quadro 2. Teste de significância, de acordo com Snedecor \& Cochran (1989), entre os modelos de capacidade de suporte de carga nas camadas de 0-3, 10-13 e 25-28 cm da área sob Latossolo Amarelo distrófico típico

\begin{tabular}{cccc}
\hline & & Parâmetro do modelo & \\
\cline { 2 - 4 } & $\mathbf{F}_{1}$ & Coeficiente angular, $\mathbf{b}$ & Intercepto, a \\
\hline $\mathrm{cm}$ & & & $\mathrm{ns}$ \\
$0-3 \times 10-13$ & $1,08 \mathrm{~ns}$ & $\mathrm{~ns}$ & $\mathrm{~ns}$ \\
$0-3 \times 25-28$ & $1,33 \mathrm{~ns}$ & $\mathrm{~ns}$ & $\mathrm{~ns}$ \\
\hline $13 \times 25-28$ & $1,23 \mathrm{~ns}$ & & \\
\hline
\end{tabular}

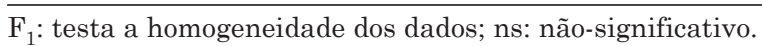

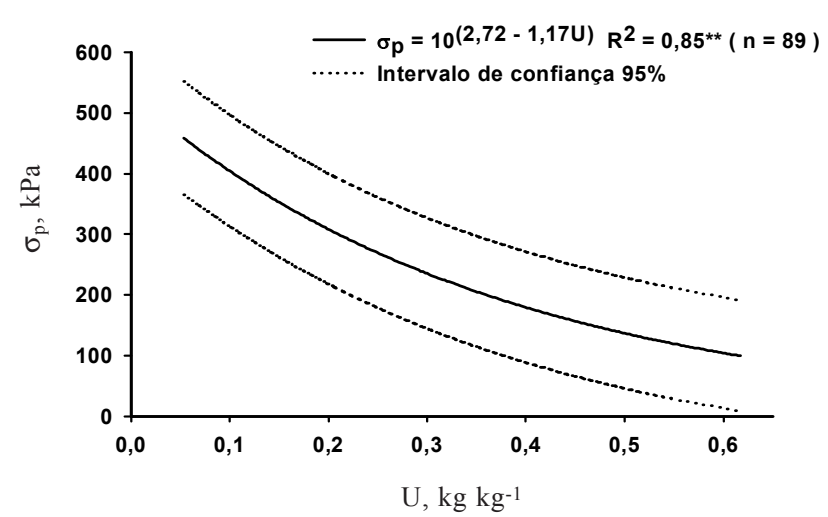

Figura 2. Modelo da capacidade de suporte de carga para o Latossolo Amarelo distrófico típico nas camadas de $0-3,10-13$ e $25-28 \mathrm{~cm}$.

serem menores do que a pressão média aplicada pelas máquinas. Por outro lado, quando o solo seca, a $\sigma_{\mathrm{p}}$ aumenta exponencialmente com a redução da umidade, aumentando sua capacidade de suporte de carga do solo, proporcionando um menor risco de ocorrência de compactação.

Considerando os critérios propostos por Dias Junior (2003), verificou-se que a Operação 1 (colhedora tracionada pelo trator marca Massey Ferguson 275) (Figura 3a) na estação seca apresentou tendência em compactar o solo na camada de $0-3 \mathrm{~cm}$, enquanto, nas camadas de 10-13 e 25-28 cm, estas operações causaram compactação do solo em 30 e $20 \%$ dos pontos amostrados, respectivamente. Tais resultados corroboram os obtidos por Castro Neto (2001), que afirmou que a camada de solo que apresenta imediatamente o efeito compactante causado pelo tráfego de um trator agrícola está situada entre a superfície e algum ponto entre $10-20 \mathrm{~cm}$ de profundidade. Estes resultados discordam daqueles obtidos por Borges et al. (2001), segundo os quais a pressão do rodado da máquina, ou mesmo de implementos agrícolas, não é transmitida a maiores profundidades.
A Operação 2 (pulverizador tracionado pelo trator Massey Ferguson 265) na estação seca apresentou tendência a compactar o solo na camada superficial de 0-3 cm, enquanto, nas camadas de 10-13 e 25$28 \mathrm{~cm}$, ocorreram compactações de 10 e $30 \%$ dos pontos amostrados, respectivamente (Figura $3 \mathrm{~b}$ ). Na estação chuvosa, o pulverizador causou, em $10 \%$ dos pontos amostrados, compactação na camada de 10-13 cm; no entanto, nas outras camadas, houve somente tendência a compactar o solo.

A Operação 3 (roçadora tracionada pelo trator Massey Ferguson 50X) na época seca (Figura 4a ) apresentou tendência a compactar o solo na camada de 0-3 cm, tendo causado compactação em $10 \%$ dos pontos amostrados na camada de $10-13 \mathrm{~cm}$ e em $50 \%$ dos pontos amostrados na camada de $25-28 \mathrm{~cm}$. $\mathrm{Na}$ estação chuvosa, este equipamento causou compactação em $60 \%$ dos pontos amostrados na camada de 0-3 cm. Essa maior compactação deveuse ao fato de ter sido o tráfego realizado em umidades acima do LP, o que conferiu ao solo, nesta umidade, baixa capacidade de suporte de carga. Esses resultados corroboram aqueles observados por Imhof et al., (2001), segundo os quais o solo, quando úmido, apresenta baixa capacidade de suporte de carga. Nas outras duas camadas, a Operação 3 apresentou tendência de compactar o solo.

A Operação 4 (granuladora tracionada pelo trator Massey Ferguson 50X) (Figura 4b), a Operação 5 (adubadora tracionada pelo trator Massey Ferguson 50X) (Figura 5a) e a Operação 6 (aplicador de herbicida tracionado pelo trator Massey Ferguson 50X) (Figura 5b), na estação chuvosa, apresentaram comportamentos semelhantes nas três profundidades estudadas, mostrando tendência de compactar o solo.

Na figura 6, são observados os efeitos cumulativos do tráfego de todos os equipamentos. Na estação seca, notou-se tendência em compactar o solo na profundidade de $0-3 \mathrm{~cm}$ tanto na linha de tráfego de todos os equipamentos quanto para a colhedora, pelo fato de sua maior bitola não coincidir com a linha de tráfego dos outros equipamentos. Para as camadas de 10-13 e 25-28 cm, 60 e $40 \%$ dos pontos 

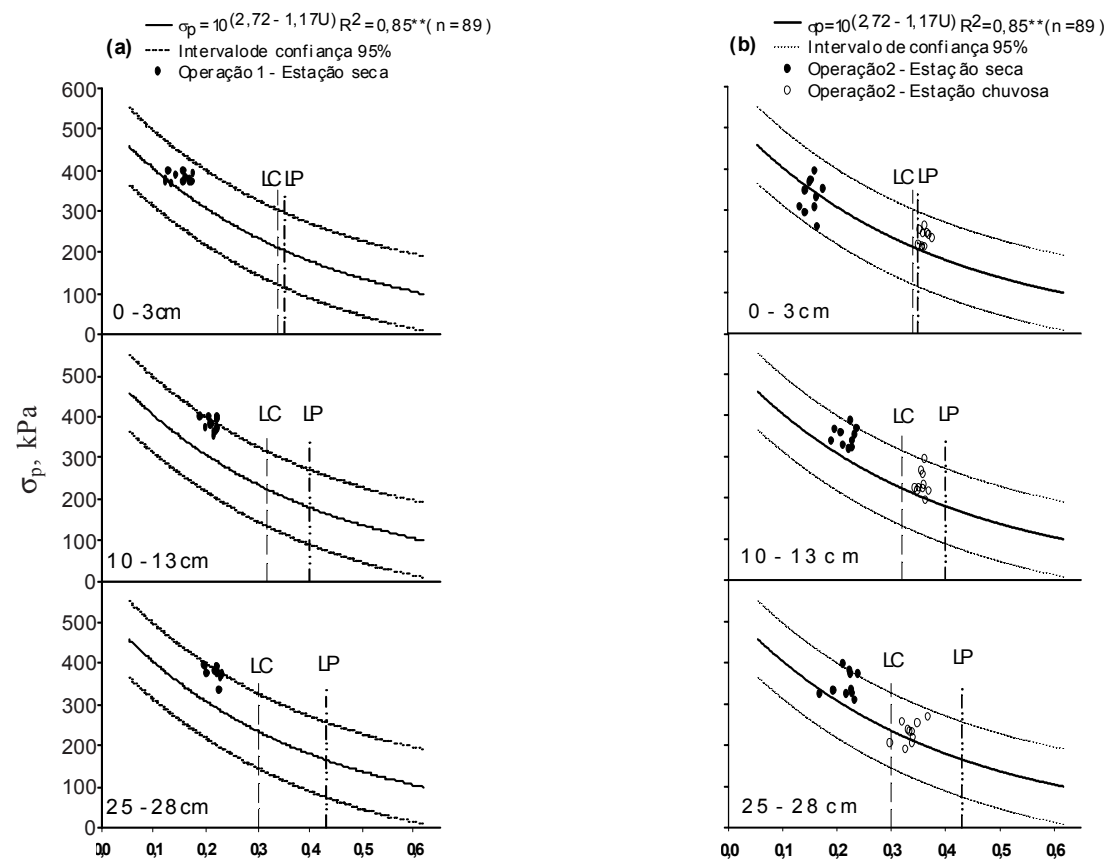

$\mathrm{U}, \mathrm{kg} \mathrm{kg}^{-1}$

Figura 3. Modelo de capacidade de suporte de carga para o Latossolo Amarelo distrófico típico nas camadas de 0-3, 10-13 e 25-28 cm com seus respectivos limites de contração (LC) e de plasticidade (LP). Os pontos representam as pressões de preconsolidação determinadas após a Operação da colhedora tracionada pelo trator Massey Ferguson 275 na estação seca (Operação1) (a), pressões de preconsolidação determinadas após as operações do pulverizador tracionado pelo trator Massey Ferguson 265 na estação seca e na chuvosa (Operação 2) (b).
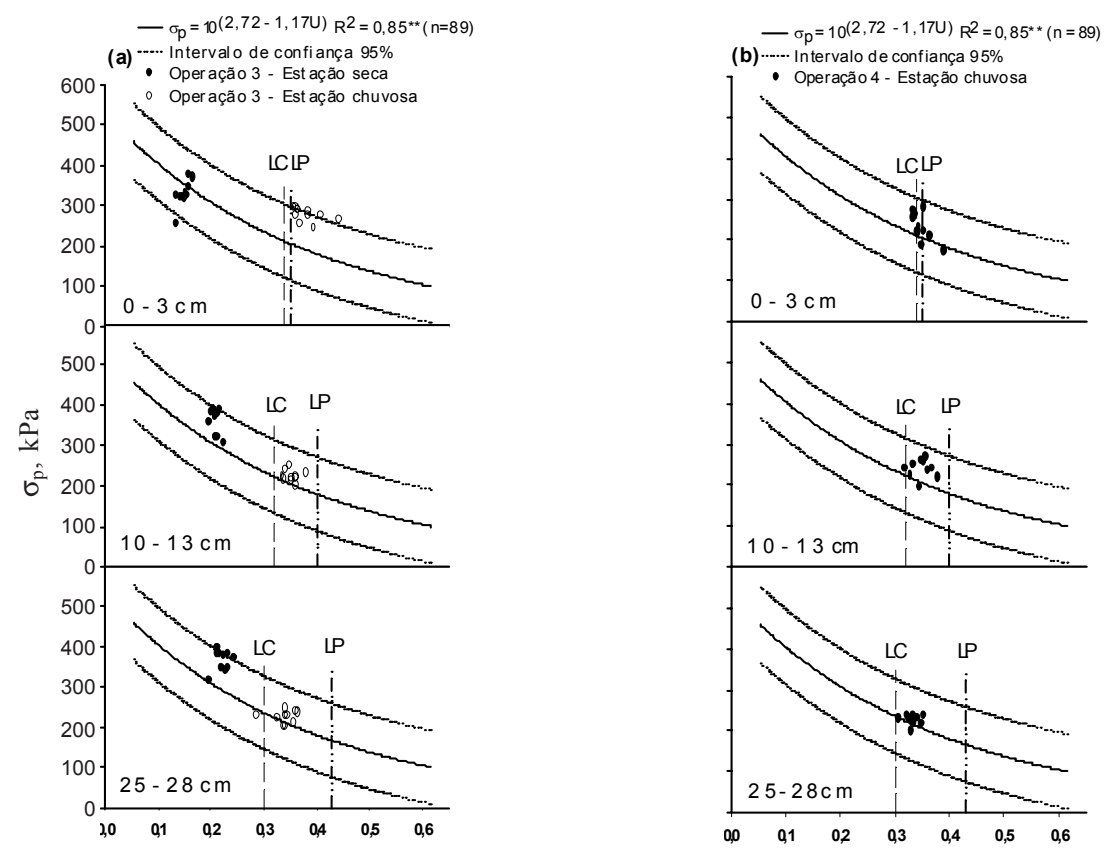

\section{$\mathrm{U}, \mathrm{kg} \mathrm{kg}^{-1}$}

Figura 4. Modelo de capacidade de suporte de carga para o Latossolo Amarelo distrófico típico nas camadas de 0-3, 10-13 e 25-28 cm com seus respectivos limites de contração (LC) e de plasticidade (LP). Os pontos representam as pressões de preconsolidação determinadas após as operações da roçadora tracionada pelo trator Massey Ferguson 50X na estação seca e tracionada pelo trator Massey Ferguson $50 \mathrm{X}$ e 265 na estação chuvosa (Operação 3) (a), as pressões de preconsolidação determinadas após as operações da granuladora tracionada pelo trator Massey Ferguson 50X na estação chuvosa (Operação 4) (b). 

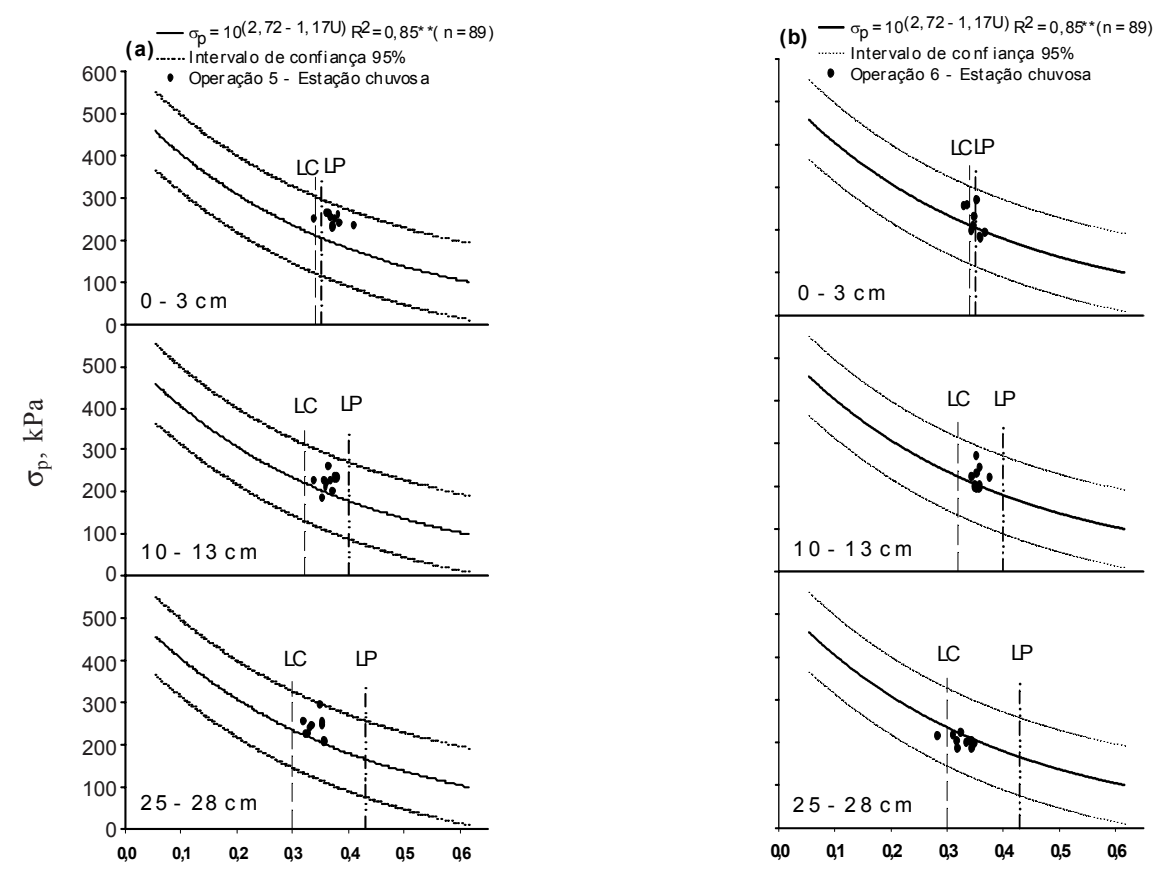

$\mathrm{U}, \mathrm{kg} \mathrm{kg}^{-1}$

Figura 5. Modelo de capacidade de suporte de carga para o Latossolo Amarelo distrófico típico nas camadas de 0-3, 10-13 e 25-28 cm com seus respectivos limites de contração (LC) e de plasticidade (LP). Os pontos representam as pressões de preconsolidação determinadas após as operações da adubadora tracionada pelo trator Massey Ferguson 50X na estação chuvosa (Operação 5) (a), as pressões de preconsolidação determinadas após a operação do aplicador de herbicida tracionado pelo trator Massey Ferguson 265 na estação chuvosa (Operação 6) (b).

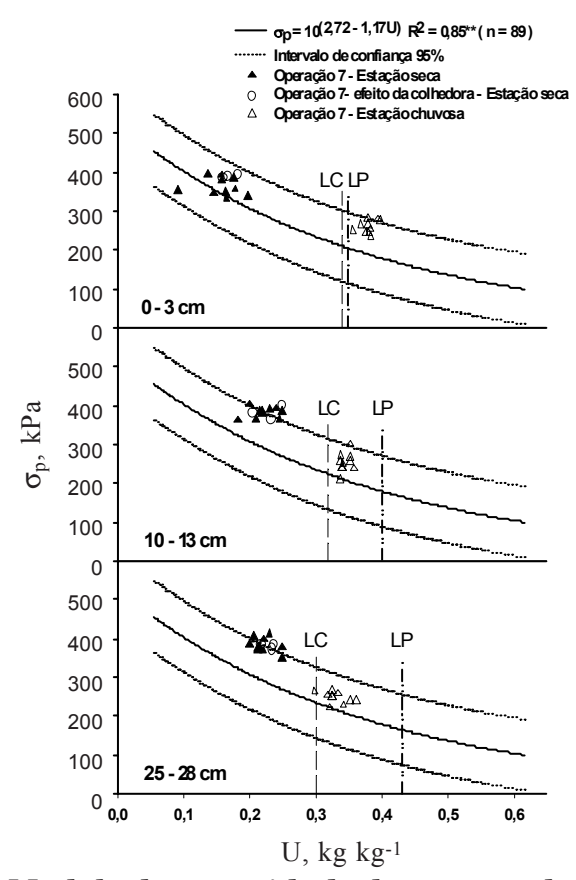

Figura 6. Modelo de capacidade de suporte de carga para o Latossolo Amarelo distrófico típico nas camadas de 0-3, 10-13 e 25-28 cm com seus respectivos limites de contração (LC) e de plasticidade (LP). Os pontos representam as pressões de preconsolidação determinadas após as operações de todos os equipamentos na estação seca e na chuvosa (Operação 7). coletados, respectivamente, apresentaram compactação. Respostas semelhantes foram encontradas por Wood et al. (1993), que verificaram que os repetidos eventos com o manejo agrícola, ou seja, o crescente número de passadas, aumentam a degradação da estrutura do solo. Na estação chuvosa, os efeitos desses equipamentos na camada de 0-3 m apresentaram compactação em $20 \%$ dos pontos coletados por terem sido as operações realizadas com umidades acima do LP, o qual representa a umidade máxima na qual as operações mecanizadas podem ser realizadas para que o solo não seja comprometido (Kondo, 1998), desde que seja observada sua capacidade de suporte de carga. Já na camada de $10-13 \mathrm{~cm}$, verificou-se que $10 \%$ das amostras estavam compactadas, enquanto, na camada de 25-28 cm, observou-se tendência a causar compactação.

Observa-se, nas figuras 3 a,b, 4 a,b, 5 a,b, 6, que as pressões de preconsolidação induzidas pelas operações mecanizadas na época chuvosa são menores do que as determinadas na época seca. Este fato evidencia, nesse caso, um alívio na resistência mecânica do solo decorrente da ação da água, corroborando os dados de Miranda et al. (2003), que revelaram o mesmo efeito em solos irrigados.

Apesar de as operações avaliadas apresentarem cargas e intensidade de tráfego diferentes, as operações 1, 2, 3 e 7 na estação seca apresentaram 
comportamentos semelhantes, provocando aumento na compactação em profundidade à medida que aumentou o número de passadas, corroborando os de Wood et al. (1993). Já na estação chuvosa, as operações 2, 3 e 7 apresentaram compactação maior na camada superficial, enquanto as operações 4,5 e 6 mantiveram comportamento semelhante.

\section{CONCLUSÕES}

1. O modelo de capacidade suporte de carga do

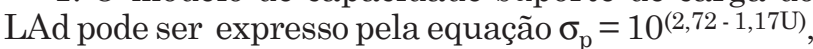
em que $\sigma_{\mathrm{p}}$ significa pressão de preconsolidação e $\vec{U}$ umidade.

2. Os efeitos das operações mecanizadas na lavoura cafeeira podem ser quantificados pelo modelo de capacidade de suporte de carga, e seu uso permite quantificar os efeitos do tráfego no manejo da lavoura cafeeira.

3. Existe redução das pressões de preconsolidação na estação chuvosa em relação à seca, indicando um aliviamento da capacidade de suporte de carga com o aumento da umidade do solo.

\section{LITERATURA CITADA}

ARVIDSSON, J. Subsoil compaction caused by heavy sugarbeet harvestes in Southern Sweden I. Soil physical properties and crop yield in six field experiments. Soil Till. Res., 60:67-78, 2001.

BLAKE, G.R. \& HARTGE, K.H. Particle density. In: KLUTE, C., ed. Methods of soil analysis. Part 1. Physical and mineralogical methods. 2.ed. Madison, American Society Agronomy, 1986. p.377-382. (Agronomy Monograph, 9)

BORGES, I.B.; LANA, R.M.Q. \& BORGES, E.N. Interação entre atributos químicos e físicos em solos de cerrado cultivados com cafeicultura no Alto Paranaíba - MG. In: SIMPÓSIO DE PESQUISA DOS CAFEEIROS DO BRASIL, 2., Vitória, 2001, Anais. Brasília, Embrapa Café, 2001. p.2555-2560

BOWLES, J.E. Engieneering properties of soils and their measurements. 3.ed. Auckland, McGraw-Hill, 1986. 218p.

CANILLAS. E.C. \& SALOKHE, V.M. A decision support system for compaction assessment in agricultural soils. Soil Till Res., 65:221-230, 2002.

ÇARMAN, K. Compaction characteristics of towed wheels on clay loam in a soil bin. Soil Till. Res., 65:37-43, 2002.

CARPENEDO, V. Compressibilidade de solos em sistemas de manejo. Porto Alegre, Universidade Federal do Rio Grande do Sul, 1994. 106p. (Tese de Doutorado)

CASTRO NETO, P. Desenvolvimento e avaliação de equipamentos e metodologia para determinação de parâmetros físicos do solo relacionados a dias trabalháveis com máquinas agrícolas. Botucatu, Universidade Estadual Paulista, 2001. 155p. (Tese de Doutorado)
CHAMEN, T.; ALAKUKKU, L.; PIRES, S.; SOMMER, C.; SPOOR, G.; TIJINK, F. \& WEISSKOPF, P. Prevention strategies for field traffic-induced subsoil compaction: A review. Part 2. Equipment and field practices. Soil Till. Res., 71:1-14, 2003.

DAY, P.R. Particle fractionation and particle size analysis. In: KLUTE, C.A., ed. Methods of soil analysis. Part 1. Physical and mineralogical methods. 2.ed. Madison, American Society Agronomy, 1986. p.545-567. (Agronomy Monograph, 9)

DIAS JUNIOR, M.S. Compactação do solo. In: NOVAIS, R.F.; ALVAREZ V., H.V. \& CHAEFER, C.E.G.R. Tópicos em ciência do solo. Viçosa, Sociedade Brasileira de Ciência do Solo, 2000. v.1. p.55-94.

DIAS JUNIOR, M.S. Compression of three soils under longterm tillage and wheel traffic. East Lansing, Michigan State University, 1994. 114p. (Tese de Doutorado)

DIAS JUNIOR, M.S. A soil mechanics approach study soil compaction In: ACHYUTHAN, H. Soil and soil physics in continental environment. Chenna, Allied Publishers Private, 2003. p.179-199.

DIAS JUNIOR, M.S.; FERREIRA, M.M.; FONSECA, S.; SILVA, A.R. \& FERREIRA, D.F. Avaliação quantitativa da sustentabilidade estrutural dos solos em sistemas florestais na região de Aracruz-ES. R. Árvore, 23:371380, 1999.

DIAS JUNIOR, M.S. \& PIERCE, F.J. Revisão de literatura. O processo de compactação do solo e sua modelagem. R. Bras. Ci. Solo, 20:175-182, 1996.

DIAS JUNIOR, M.S. \& PIERCE, F.J. A simple procedure for estimating preconsolidation pressure from soil compression curves. Soil Technol., 8:139-151, 1995.

EMPRESA BRASILEIRA DE PESQUISA AGROPECUÁRIA EMBRAPA. Centro Nacional de Pesquisas de Solo. Sistema brasileiro de classificação de solos. Rio de Janeiro, 1999. 412p.

GUIMARÃES, E.C. Variabilidade espacial de atributos de um Latossolo Vermelho-Escuro textura argilosa da região do cerrado, submetido ao plantio convencional. Campinas, Universidade Estadual de Campinas, 2000. 89p. (Tese de Doutorado)

GYSI, M. Compaction of a Eutric Cambisol under heavy wheel traffic Switzerland: Field data and a critical state soil mecchanics model approach. Soil Till. Res., 61:133-142, 2001.

HILLEL, D. Introduction to soil physics. San Diego, Academic Press, 1982. 365p.

IMHOF, S.; SILVA, A.P.; DIAS JUNIOR, M.S. \& TORMENA, C.A. Quantificação de pressões críticas para o crescimento das plantas. R. Bras. Ci. Solo, 25:11-18, 2001.

ISHAQ, M.; IBRAHIM, M.; HASSAN, A.; SAEED, M. \& LAL, R. Subsoil compaction effects on crops in Punjab, Pakistan: II. Root growth and nutrient uptake of wheat and sorghum. Soil Till. Res., 60:153-161, 2001.

KONDO, M.K. Compressibilidade de três Latossolos sob diferentes usos. Lavras, Universidade Federal de Lavras, 1998. 95p. (Tese de Mestrado) 
LARSON, W.E.; BLAKE, G.R.; ALLMARAS, R.R. VOORHEES, W.B. \& GUPTA, S.C. Mechanics and related processes in structured agricultural soils. The Netherlands, Kluwer Academic Publishers, 1989. 273p. (NATO Applied Science, 172)

MANTOVANI, E.C. Compactação do solo. Inf. Agropec., 13:52 55,1987 .

MIRANDA, E.E.V.; DIAS JUNIOR, M.S.; GUIMARÃES, P.T.G.; PINTO, J.A.O.; ARAÚJO JUNIOR, C.F. \& LASMAR JUNIOR, E. Efeito do manejo e do tráfego nos modelos de sustentabilidade da estrutura de um Latossolo Vermelho cultivado com cafeeiros. Ci. Agrotec., 1506-1515, 2003. (Edição especial)

NEVENS, F. \& REHEUL, D. The consequences of wheelinduced soil compaction and sub soiling for silage maize on a sandy loam soil in Belgium. Soil Till. Res., 70:175$184,2003$.

NOVAIS, R.F. \& SMYTH, T.J. Fósforo em solo e plantas em condições tropicais. Viçosa, Universidade Federal Viçosa, 1999. 399p.

OLIVEIRA, G.C.; DIAS JUNIOR, M.S.; RESCK, D.V.S. \& CURI, N. Alterações estruturais e comportamento compressivo de um Latossolo Vermelho distrófico argiloso sob diferentes sistemas de uso e manejo. Pesq. Agropec. Bras., 38:47-50, 2003.

PYTKA, J. Load effect upon soil stress and deformation state in structured and disturbed sandy loam for two tillage treatments. Soil Till. Res., 59:13-25, 2001.

RAIJ, B. van \& GUAGGIO, J.A. Métodos de análise de solo para fins de fertilidade. Campinas, Instituto Agronômico, 1983. 16p. (Circular, 63)

RIBEIRO, M.A.V. Resposta da soja e do eucalipto a fósforo em solos de diferentes texturas, níveis de densidade e de umidade. Lavras, Universidade Federal de Lavras, 1999. 71p. (Tese de Doutorado)
SERVADIO, P ; MARSILI, A ; PAGLIAI, M.; PELLEGRINI, S. \& VIGNOZZI, N. Effects on some clay soil qualities following the passage of rubber-tracked and wheeled tractors in central Italy. Soil Till. Res., 61:143-155, 2001.

SILVA, R.B.; LIMA, J.M. \& DIAS JUNIOR, M.S. Efeito da adsorção de fosfato em parâmetros físicos e na compressibilidade de solos tropicais. R. Bras. Ci. Solo, 23:219-226, 1999.

SILVA, R.V.; REINERT, D.J.; REICHERT, J.M. \& SOARES, J.M. Fatores controladores da compressibilidade de um Argissolo Vermelho-Amarelo distrófico arênico e de um Latossolo Vermelho distrófico típico. I - Estado inicial de compactação. R. Bras. Ci. Solo, 26:1-8, 2002.

SNEDECOR, G.W. \& COCHRAN, W.G. Statistical methods. 8.ed. Ames, Iowa State University Press, 1989. 503p.

SOWERS, G.F. Consistency. In: BLACK, C.A., ed. Methods of soil analisys - Physical and mineralogical properties including statistics of measurement and sampling. Madison, American Society of Agronomy, 1965. pt: 1, p.391-399.

TAYLOR, H.M. Effects of soil strength on seedling mergence, root growth and crop yield. In: BARNES, K.K.; CARLETON, W.M.; TAYLOR, H.M.; THROCKMORTON, R.I. \& van den BERG, G.E. Compaction of agricultural soils. St. Joseph, ASAE, 1971. p.292-305. (Monograph)

TEODORO, R.E.F.; MELO, B.; SEVERINO, G.M.; FERNANDES, D.L.; FERREIRA NETO, J.G. \& MARCUZZO, K.V. Avaliação de diferentes lâminas de irrigação do cafeeiro no cerrado mineiro. In: SIMPÓSIO DE PESQUISA DOS CAFÉS DO BRASIL, 3., Porto Seguro, 2003. Anais. Brasília, Embrapa Café, 2003. p.114.

WILLIAMSON, J.R. \& NIELSEN, W.A. The effect of soil compaction, profile disturbance and fertilizer application on the growth of eucalypt seedlings in two glasshouse studies. Soil Till. Res., 71:95-107, 2003.

WOOD, R.K.; REEDER, R.C.; MORGAN, M.T. \& HOLMES, R.G. Soil physics properties as affected grain cart traffic. Trans. Am. Soc. Agric. Eng., 36:11-14, 1993. 